\title{
ZERO-HOPF BIFURCATIONS IN THREE-DIMENSIONAL CHAOTIC SYSTEMS WITH ONE STABLE EQUILIBRIUM
}

\author{
JAUME LLIBRE ${ }^{1}$, MARCELO MESSIAS ${ }^{2}$ AND ALISSON C. REINOL ${ }^{3}$
}

\begin{abstract}
In (Molaie et al., Int J Bifurcat Chaos 23 (2013) 1350188) the authors provided the expressions of twenty three quadratic differential systems in $\mathbb{R}^{3}$ with the unusual feature of having chaotic dynamics coexisting with one stable equilibrium point. In this paper we consider twenty three classes of quadratic differential systems in $\mathbb{R}^{3}$ depending on a real parameter $a$ which, for $a=1$, coincide with the differential systems given by Molaie et al. We study the dynamics and bifurcations of these classes of differential systems by varying the parameter value $a$. We prove that, for $a=0$ all the twenty three considered systems have a zero-Hopf equilibrium point located at the origin. For $a>0$ small enough, three periodic orbits bifurcate from the origin: one of them unstable and the other two forming a pair of saddle type periodic orbits. Furthermore, we show numerically that the hidden chaotic attractors which exist for these systems when $a=1$ (already described by Molaie et al.) are obtained by period-doubling route to chaos.
\end{abstract}

\section{Introduction AND StATEMENT of the main RESUlts}

The interest in finding and studying chaotic differential systems has aroused considerably since Lorenz reported in 1963 a chaotic attractor in a threedimensional autonomous system [15, 20]. Recently, chaotic attractors have been categorized in two classes: self-excited and hidden attractors. Whereas a self-excited attractor has a basin of attraction that overlaps with the neighborhood of an unstable equilibrium point, a hidden attractor has a basin of attraction which does not intersect with small neighborhoods of any equilibrium point $[11,12,13]$.

The well-known Lorenz system [15], Rössler system [18] and Chua system [6] are examples of chaotic systems with self-excited attractors. Hidden attractors can be found in chaotic differential systems without equilibria $[8,14,22]$, with only stable equilibrium points $[10,17,23,24,25]$ or with an infinite number of equilibria (curves, surfaces) [7,9] and there is little knowledge about their formation, hence analytical proofs of their existence is yet needed. From the point of view of applications, hidden attractors are potentially important in engineering applications $[1,2]$, chaos-based secure

Key words and phrases. Zero-Hopf bifurcation, periodic orbits, period-doubling route to chaos, hidden chaotic attractors. 
communication [21] and image encryption [5] and the number of applications is increasing as studies on this type of attractors progress.

In [17], using the Routh-Hurwitz stability criterion and a systematic computer search, the authors provided the expressions of twenty three quadratic differential systems in $\mathbb{R}^{3}$ presenting chaotic behavior which coexist with only one stable equilibrium point and, therefore, have a hidden chaotic attractor. In order to contribute to the understanding of the dynamics of these unusual types of quadratic systems, in this paper we consider twenty three classes of quadratic differential systems in $\mathbb{R}^{3}$, depending on a real parameter $a$, which we denote by the $\mathrm{SE} a_{1}-\mathrm{SE} a_{23}$ systems, which coincide with the differential systems studied in [17] when $a=1$. In this way, the one-parameter systems considered here, given in Tables 1, 2 and 3, present chaotic behavior with only one stable equilibrium point when $a=1$. Furthermore, for $a=0$ there is a straight line passing by the origin, filled up by equilibrium points, and the origin is a zero-Hopf equilibrium, for all the 23 systems considered here.

The main result of this paper is the following.

Theorem 1. For $a=0$, systems $S E a_{1}$ to $S E a_{23}$ have one curve of equilibrium points given by a straight line containing the origin. In a neighborhood of the origin, the singularities form two branches of foci with opposite stability connected by a zero-Hopf equilibrium point at the origin. For a $>0$ small enough, the $S E a_{1}-S E a_{23}$ systems have three periodic orbits which bifurcate from the zero-Hopf equilibrium at the origin: one of them is unstable and the other two form a pair of saddle type periodic orbits.

Theorem 1 is proved in Section 3. We recall that an (isolated) equilibrium point of a differential system in $\mathbb{R}^{3}$ is called a zero-Hopf equilibrium point if the Jacobian matrix of the system at this point has a zero and a pair of purely imaginary eigenvalues. As for $a=0$ the origin of the $\mathrm{SE} a_{1}-\mathrm{SE} a_{23}$ systems is a non-isolated equilibrium point with eigenvalues 0 and $\pm i$, we call it a non-isolated zero-Hopf equilibrium point.

The existence of periodic orbits plays an important role in the formation of chaotic attractors. Indeed some known routes to chaos are characterized by the existence of this kind of orbits. In [17] the authors stated that the differential systems studied by them $\left(\mathrm{SE} a_{1}-\mathrm{SE}_{23}\right.$ systems in Table 1 of [17]) appear to approach chaotic regime through a succession of perioddoubling bifurcations of periodic orbits and, as an example, they numerically showed this phenomenon for their $\mathrm{SE}_{3}$ system (which corresponds to our $\mathrm{SE} a_{3}$ system with $a=1$ ), taking the third equation as $\dot{z}=-\alpha x-y-4 z+$ $y^{2}+x y$ and varying parameter $\alpha$ from 3.3 to 3.4 (see Fig. 2 of [17]).

Here, by using numerical simulations, we show that a cascade of perioddoubling bifurcations also occur for the classes of differential systems $\mathrm{SE} a_{1}$ - $\mathrm{SE} a_{23}$, as the parameter $a$ is varied near $a=1$. We summarize it in the following result. 


\begin{tabular}{|c|c|c|c|}
\hline Model & Equat & tions & $\begin{array}{l}\text { Equilibrium } \\
\text { point }\end{array}$ \\
\hline $\mathrm{SE} a_{1}$ & $\begin{array}{l}\dot{x}= \\
\dot{y}= \\
\dot{z}=\end{array}$ & $\begin{array}{l}y \\
z \\
-a x+(0.4 a-1) y-2 a z+z^{2}-0.4 x y \\
-0.5(a-1) x z+a^{2}(a-1)\end{array}$ & $\left(a^{2}-a, 0,0\right)$ \\
\hline $\mathrm{SE} a_{2}$ & $\begin{array}{l}\dot{x}= \\
\dot{y}= \\
\dot{z}=\end{array}$ & $\begin{array}{l}y \\
z \\
-0.5 a x-y-0.55 a z-1.2 z^{2} \\
-0.1(11-a) x z-y z-0.5 a^{2}(a-1) .\end{array}$ & $\left(a-a^{2}, 0,0\right)$ \\
\hline $\mathrm{SE} a_{3}$ & $\begin{array}{l}\dot{x}= \\
\dot{y}= \\
\dot{z}=\end{array}$ & $\begin{array}{l}y, \\
z, \\
-3.4 a x-y-4 a z+y^{2}+x y \\
-0.5(a-1) x z+a^{2}(a-1)\end{array}$ & $\left(\frac{a^{2}-a}{3.4}, 0,0\right)$ \\
\hline $\mathrm{SE} a_{4}$ & $\begin{array}{l}\dot{x}= \\
\dot{y}= \\
\dot{z}=\end{array}$ & $\begin{array}{l}y \\
z \\
-a x+(a-1) y-1.7 a z+y^{2}+0.6 x y \\
-0.5(a-1) x z-a^{2}\end{array}$ & $(-a, 0,0)$ \\
\hline $\mathrm{SE} a_{5}$ & $\begin{array}{l}\dot{x}= \\
\dot{y}= \\
\dot{z}=\end{array}$ & $\begin{array}{l}y, \\
z, \\
-a x+(a-1) y-a z-2(a-1) y^{2}-z^{2} \\
+0.4 x y-0.5(a-1) x z-2.7 a^{2} .\end{array}$ & $(-2.7 a, 0,0)$ \\
\hline $\mathrm{SE} a_{6}$ & $\begin{array}{l}\dot{x}= \\
\dot{y}= \\
\dot{z}=\end{array}$ & $\begin{array}{l}y, \\
z, \\
-a x+(a-1) y-5(a-1) y^{2}-2.9 z^{2} \\
+x y+1.1 x z-a^{2} .\end{array}$ & $(-a, 0,0)$ \\
\hline
\end{tabular}

TABlE 1. The $\mathrm{SE} a_{1}-\mathrm{SE} a_{6}$ systems studied in this paper and their stable equilibrium point for $a>0$. When $a=1$ these systems are the ones provided in [17].

Conjecture. The hidden chaotic attractors which exist for $a=1$ in the $S E a_{1}-S E a_{23}$ systems displayed in Tables 1, 2 and 3, are formed by the occurrence of period-doubling bifurcations of periodic orbits, as the parameter $a$ is varied.

In order to illustrate the result stated above, in Section 4, we numerically study the formation of hidden chaotic attractors in the $\mathrm{SE} a_{1}$ and $\mathrm{SE} a_{11}$ systems. The dynamical behavior is quite similar for the other systems .

Based on the results described above, we conjecture that the hidden chaotic attractors of $\mathrm{SE} a_{i}$ systems with $a=1$, for $i=1, \ldots, 23$, are created by a cascade of period doubling bifurcations which start at one of the periodic orbits bifurcated from the zero-Hopf equilibrium point at the origin. Due to the instability of these periodic orbits, the numerical study of their 


\begin{tabular}{|c|c|c|c|}
\hline Model & Equa & tions & $\begin{array}{l}\text { Equilibrium } \\
\text { point }\end{array}$ \\
\hline $\mathrm{SE}_{7}$ & $\begin{array}{l}\dot{x}= \\
\dot{y}= \\
\dot{z}=\end{array}$ & $\begin{array}{l}y, \\
-x+y z, \\
-2 a z+(1-a) x^{2}-8 x y+x z-a^{2} .\end{array}$ & $(0,0,-0.5 a)$ \\
\hline $\mathrm{SE} a_{8}$ & $\begin{array}{l}\dot{x}= \\
\dot{y}= \\
\dot{z}=\end{array}$ & $\begin{array}{l}y, \\
-x+y z, \\
-a z-0.7 x^{2}+y^{2}-0.1 a^{2}\end{array}$ & $(0,0,-0.1 a)$ \\
\hline $\mathrm{SE} a_{9}$ & $\begin{array}{l}\dot{x}= \\
\dot{y}= \\
\dot{z}=\end{array}$ & $\begin{array}{l}y, \\
-x+y z, \\
2 a x-2 a z+y^{2}-0.3 a^{2} .\end{array}$ & $(0,0,-0.15 a)$ \\
\hline $\mathrm{SE} a_{10}$ & $\begin{array}{l}\dot{x}= \\
\dot{y}= \\
\dot{z}=\end{array}$ & $\begin{array}{l}y, \\
-x+y z \\
a x-0.3 a y-2 a z+(1-a) x^{2}+x z-0.1 a^{2} .\end{array}$ & $(0,0,-0.05 a)$ \\
\hline $\mathrm{SE} a_{11}$ & $\begin{array}{l}\dot{x}= \\
\dot{y}= \\
\dot{z}=\end{array}$ & $\begin{array}{l}y, \\
-x+y z, \\
-a y-12 a z+x^{2}+9 x z-a^{2} .\end{array}$ & $\left(0,0,-\frac{a}{12}\right)$ \\
\hline $\mathrm{SE} a_{12}$ & $\begin{array}{l}\dot{x}= \\
\dot{y}= \\
\dot{z}=\end{array}$ & $\begin{array}{l}y, \\
-x+y z, \\
-66 a z+y^{2}+35 x z-a^{2} .\end{array}$ & $\left(0,0,-\frac{a}{66}\right)$ \\
\hline $\mathrm{SE} a_{13}$ & $\begin{array}{l}\dot{x}= \\
\dot{y}= \\
\dot{z}=\end{array}$ & $\begin{array}{l}y, \\
-x+y z, \\
-4.9 a z+0.4 y^{2}+x y-a^{2}\end{array}$ & $\left(0,0,-\frac{a}{4.9}\right)$ \\
\hline $\mathrm{SE} a_{14}$ & $\begin{array}{l}\dot{x}= \\
\dot{y}= \\
\dot{z}=\end{array}$ & $\begin{array}{l}z \\
x+z \\
(a-1) x-a y-5(a-1) x^{2}-3 z^{2}+x y \\
+(2-a) y z-0.7 a^{2}\end{array}$ & $(0,-0.7 a, 0)$ \\
\hline $\mathrm{SE} a_{15}$ & $\begin{array}{l}\dot{x}= \\
\dot{y}= \\
\dot{z}=\end{array}$ & $\begin{array}{l}-z \\
x-z \\
(1-a) x+0.9 a y+0.2 x^{2}+2(a-1) z^{2} \\
+x z+y z+a^{2}\end{array}$ & $\left(0,-\frac{a}{0.9}, 0\right)$ \\
\hline $\mathrm{SE} a_{16}$ & $\begin{array}{l}\dot{x}= \\
\dot{y}= \\
\dot{z}=\end{array}$ & $\begin{array}{l}-z \\
-x+z \\
(1-a) x-7 a y-1.4 a(10-9 a) z \\
+(3 a-2) x^{2}+x z-y z+a^{2}(a-1)\end{array}$ & $\left(0, \frac{a^{2}-a}{7}, 0\right)$ \\
\hline $\mathrm{SE} a_{17}$ & $\begin{array}{l}\dot{x}= \\
\dot{y}= \\
\dot{z}=\end{array}$ & $\begin{array}{l}z \\
x-y \\
-(1.1 a+2) x-2(a-1) y-(a-1) z \\
-0.3(50-49 a) x z+0.2 y z+0.57 a^{2}\end{array}$ & $\left(\frac{0.57 a}{3.1}, \frac{0.57 a}{3.1}, 0\right)$ \\
\hline $\mathrm{SE} a_{18}$ & $\begin{array}{l}\dot{x}= \\
\dot{y}= \\
\dot{z}=\end{array}$ & $\begin{array}{l}z, \\
-y+z \\
-2.1 a x+2(a-1) y-(1.1 a-1) z-(2 a-1) y^{2} \\
+0.11(100-99 a) x z+0.5 y z+a^{2}(a-1) .\end{array}$ & $\left(\frac{a^{2}-a}{2.1}, 0,0\right)$ \\
\hline
\end{tabular}

TABlE 2. The SE $a_{7}-\mathrm{SE} a_{18}$ systems studied in this paper and their stable equilibrium point for $a>0$. For $a=1$ these systems are the ones provided in [17]. 


\begin{tabular}{|c|c|c|c|}
\hline Model & Equat & tions & $\begin{array}{l}\text { Equilibrium } \\
\text { point }\end{array}$ \\
\hline $\mathrm{SE} a_{19}$ & $\begin{array}{l}\dot{x}= \\
\dot{y}= \\
\dot{z}=\end{array}$ & $\begin{array}{l}z \\
-y+z \\
-a x+2(a-1) y-(a-1) z-3(a-1) y^{2} \\
-2 x y+1.7 x z-0.3 a^{2}\end{array}$ & $(-0.3 a, 0,0)$ \\
\hline $\mathrm{SE} a_{20}$ & $\begin{array}{l}\dot{x}= \\
\dot{y}= \\
\dot{z}=\end{array}$ & $\begin{array}{l}z \\
-y-z \\
-11 a x+2 y+(1-a) z-2 y^{2}-(51 a-50) z^{2} \\
-60(a-1) x y-120(1-a) x z-y z+a^{2}(a-1)\end{array}$ & $\left(\frac{a^{2}-a}{11}, 0,0\right)$ \\
\hline $\mathrm{SE} a_{21}$ & $\begin{array}{l}\dot{x}= \\
\dot{y}= \\
\dot{z}=\end{array}$ & $\begin{array}{l}z, \\
-y-z, \\
-7.1 a x+(2-a) y+(1-a) z-2 y^{2} \\
+(50 a-49) x z-y z-a^{2}(a-1) .\end{array}$ & $\left(\frac{a-a^{2}}{7.1}, 0,0\right)$ \\
\hline $\mathrm{SE} a_{22}$ & $\begin{array}{l}\dot{x}= \\
\dot{y}= \\
\dot{z}=\end{array}$ & $\begin{array}{l}z, \\
-y-z, \\
-6 a x-2(a-1) y-(a-1) z-2(2 a-1) y^{2} \\
+(50-49 a) x z-y z-0.9 a^{2} .\end{array}$ & $(-0.15 a, 0,0)$ \\
\hline $\mathrm{SE} a_{23}$ & $\begin{array}{l}\dot{x}= \\
\dot{y}= \\
\dot{z}=\end{array}$ & $\begin{array}{l}-z \\
-y-z \\
4 a x-2(a-1) y-(a-1) z-0.2 z^{2}+x y \\
+10(a-1) x z-2 a^{2}\end{array}$ & $(0.5 a, 0,0)$ \\
\hline
\end{tabular}

TABlE 3. The SE $a_{19}-\mathrm{SE} a_{23}$ systems studied in this paper and their stable equilibrium point for $a>0$. For $a=1$ these systems are the ones provided in [17].

continuation, from $a=0$ to $a=1$ is very difficult. However, there is no the occurrence of other Hopf like bifurcations as the parameter $a$ varies between 0 and 1 , because systems $\mathrm{SE} a_{i}$ have only one equilibrium point whose stability does not change. This fact led us to formulate such a conjecture.

This paper is devoted to prove Theorem 1 and it is organized as follows. In Section 2 we present some basic aspects of the averaging theory of first order, which we shall use to prove Theorem 1 in Section 3. The formation of the hidden chaotic attractors of $\mathrm{SE} a_{1}$ and $\mathrm{SE} a_{11}$ systems with $a=1$ are numerically studied in Section 4 and these numerical results motivate the conjecture. Finally in Section 5 we give some concluding remarks.

\section{Averaging theORY OF FIRST ORDER}

For the sake of completeness and to fix the notation which will be used ahead, in this section we present the resuelt from averaging theory that we shall use for proving Theorem 1, its proof can be found in [19]. 
Consider the initial value problems

$$
\dot{\mathbf{x}}=\varepsilon F_{1}(t, \mathbf{x})+\varepsilon^{2} F_{2}(t, \mathbf{x}, \varepsilon), \quad \mathbf{x}(0)=\mathbf{x}_{0},
$$

and

$$
\dot{\mathbf{y}}=\varepsilon g(\mathbf{y}), \quad \mathbf{y}(0)=\mathbf{x}_{0},
$$

where $\mathbf{x}=\mathbf{x}(t)$ and $\mathbf{y}=\mathbf{y}(t)$ are defined in some open subset $\Omega \subset \mathbb{R}^{n}$ for $t \in[0, \infty), \mathbf{x}_{0}$ is a point of $\Omega$ and $\varepsilon \in\left(0, \varepsilon_{0}\right]$, for some fixed $\varepsilon_{0}>0$ small enough. Suppose that $F_{1}$ and $F_{2}$ are periodic functions of period $T$ in the variable $t$, and set

$$
g(\mathbf{y})=\frac{1}{T} \int_{0}^{T} F_{1}(t, \mathbf{y}) d t .
$$

Assume that $D_{\mathbf{x}} g$ and $D_{\mathbf{x x}} g$ are all the first and second derivatives of $g$ respectively. Under these assumptions, we have the following result.

Theorem 2. Let $F_{1}, D_{\mathbf{x}} F_{1}, D_{\mathbf{x x}} F_{1}$ and $D_{\mathbf{x}} F_{2}$ be continuous and bounded by a constant, which does not depend on $\varepsilon$, in $[0, \infty) \times \Omega \times\left(0, \varepsilon_{0}\right]$ and suppose that $\mathbf{y}(t) \in \Omega$ for $t \in[0,1 / \varepsilon]$. Then, the following statements hold.

1. For $t \in[0,1 / \varepsilon]$, we have $\mathbf{x}(t)-\mathbf{y}(t)=\mathcal{O}(\varepsilon)$ as $\varepsilon \rightarrow 0$.

2. If $p \neq 0$ is an equilibrium point of system (2) such that $\operatorname{det}\left[D_{\mathbf{y}} g(p)\right] \neq$ 0 , then system (1) has a periodic solution $\phi(t, \varepsilon)$ of period $T$, which is close to $p$ and such that $\phi(0, \varepsilon)-p=\mathcal{O}(\varepsilon)$ as $\varepsilon \rightarrow 0$.

3. The stability of the periodic solution $\phi(t, \varepsilon)$ is given by the stability of the equilibrium point $p$.

\section{Proof of Theorem 1}

Consider the $\mathrm{SE} a_{1}$ system given in Table 1 . For $a=0$ the $x$-axis is filled up of equilibrium points. The linear part of $\mathrm{SE} a_{1}$ system at these equilibrium points are

$$
\lambda_{1}=0 \quad \text { and } \quad \lambda_{2,3}=\frac{x}{4} \pm \frac{\sqrt{25 x^{2}-160 x-400}}{20} .
$$

Note that, in a neighborhood of the origin, the $x$-axis is formed by two branches of foci with opposite stability connected by the origin, which is a non-isolated zero-Hopf equilibrium point with eigenvalues $\lambda_{1}=0$ and $\lambda_{2,3}= \pm i$.

Now, using Theorem 2, we prove that three periodic orbits bifurcate from the origin for $a>0$ small enough. In order to put the $\mathrm{SE} a_{1}$ system in the appropriate normal form to apply the averaging theorem, first consider the linear change of variables

$$
(x, y, z) \rightarrow(u, v, w), \quad \text { where } x=u+w, y=v \text { and } z=-u .
$$

In the new variables $(u, v, w)$ the $\mathrm{SE} a_{1}$ system becomes

$$
\begin{aligned}
& \dot{u}=-a u+(1-0.4 a) v+a w+F_{1}(u, v, w), \\
& \dot{v}=-u, \\
& \dot{w}=a u+0.4 a v-a w+F_{2}(u, v, w),
\end{aligned}
$$


where

$$
F_{1}(u, v, w)=-\frac{1}{2}(1+a) u^{2}+\frac{2}{5} u v+\frac{1}{2}(1-a) u w+\frac{2}{5} v w-a^{2}(a-1),
$$

and $F_{2}(u, v, w)=-F_{1}(u, v, w)$. Note that the linear part of system (3) at the origin when $a=0$ is in the real Jordan normal form, that is in the form

$$
\left(\begin{array}{ccc}
0 & 1 & 0 \\
-1 & 0 & 0 \\
0 & 0 & 0
\end{array}\right) \text {. }
$$

Now write system (3) in cylindrical coordinates $(r, \theta, w)$ doing the change of variables $u=r \cos \theta, v=r \sin \theta$. Then system (3) becomes

$$
\begin{aligned}
& \dot{r}=A_{1}(\theta, w) r^{2}+A_{2}(\theta, w) r+A_{3}(\theta, w), \\
& \dot{\theta}=-1+B_{1}(\theta, w) r+B_{2}(\theta, w)+\frac{1}{r} B_{3}(\theta, w), \\
& \dot{w}=C_{1}(\theta, w) r^{2}+C_{2}(\theta, w) r+C_{3}(\theta, w),
\end{aligned}
$$

where

$$
\begin{aligned}
& C_{1}(\theta, w)=\frac{1}{2}(a+1) \cos ^{2} \theta-\frac{2}{5} \sin \theta \cos \theta \\
& C_{2}(\theta, w)=\frac{1}{2}((w+2) a-w) \cos \theta+\frac{2}{5}(a-w) \sin \theta, \\
& C_{3}(\theta, w)=a\left(a^{2}-a-w\right), \\
& A_{i}(\theta, w)=-C_{i}(\theta, w) \cos \theta \text { and } B_{i}(\theta, w)=C_{i}(\theta, w) \sin \theta
\end{aligned}
$$

for $i=1,2,3$. Introduce the variable $\varepsilon>0$ into system (4) by considering $a=\varepsilon$ and doing a rescaling of the variables through the change of coordinates

$$
(r, \theta, w) \rightarrow(R, \theta, W), \quad \text { where } r=\varepsilon R \text { and } w=\varepsilon W .
$$

Then, taking $\theta$ as the new independent variable and doing the Taylor expansion in the variable $\varepsilon$ at $\varepsilon=0$ up to order 2 we get

$$
\begin{aligned}
& \frac{d R}{d \theta}=F_{11}(\theta, R, W) \varepsilon+\mathcal{O}\left(\varepsilon^{2}\right), \\
& \frac{d W}{d \theta}=F_{21}(\theta, R, W) \varepsilon+\mathcal{O}\left(\varepsilon^{2}\right),
\end{aligned}
$$

where

$$
\begin{aligned}
F_{11}(\theta, R, W)= & \frac{1}{2} \cos \theta\left[R^{2} \cos ^{2} \theta-\left(0.8 R^{2} \sin \theta+R(W-2)\right] \cos \theta\right. \\
& -0.8 R(W-1) \sin \theta-2(W+1)) \\
F_{21}(\theta, R, W)= & -\frac{1}{2} R^{2} \cos ^{2} \theta-\frac{1}{2}\left[\left(-0.8 R^{2} \sin \theta+R(2-W)\right] \cos \theta\right. \\
& +0.4 R(W-1) \sin \theta+W+1 .
\end{aligned}
$$

Using the notation of Theorem 2, consider

$$
\mathbf{x}=\mathbf{y}=\left(\begin{array}{c}
R \\
W
\end{array}\right), \quad t=\theta, \quad T=2 \pi, \quad F_{1}(\theta, \mathbf{x})=\left(\begin{array}{c}
F_{11}(\theta, \mathbf{x}) \\
F_{21}(\theta, \mathbf{x})
\end{array}\right) .
$$


In this way we have

$$
g(\mathbf{y})=\frac{1}{2 \pi} \int_{0}^{2 \pi} F_{1}(\theta, \mathbf{y}) d \theta=-\frac{1}{4}\left(\begin{array}{c}
R(W-2) \\
R^{2}-4(W+1)
\end{array}\right) .
$$

Hence $g(\mathbf{y})=0$ has the real solutions

$$
p_{1}=\left(R_{1}, W_{1}\right)=(0,-1) \quad \text { and } \quad p_{2,3}=\left(R_{2,3}, W_{2,3}\right)=( \pm 2 \sqrt{3}, 2),
$$

which satisfy $\operatorname{det}\left[D_{\mathbf{y}} g\left(p_{1}\right)\right]=3 / 4 \neq 0$ and $\operatorname{det}\left[D_{\mathbf{y}} g\left(p_{2}\right)\right]=\operatorname{det}\left[D_{\mathbf{y}} g\left(p_{3}\right)\right]=$ $-3 / 2 \neq 0$. Then, by Theorem 2 , for $\varepsilon>0$ sufficiently small, the $\mathrm{SE} a_{1}$ system has the periodic solutions $\phi_{i}(\theta, \varepsilon)=\left(R_{i}(\theta, \varepsilon), Z_{i}(\theta, \varepsilon)\right)$, for $i=1,2,3$, such that $\phi_{i}(0, \varepsilon) \rightarrow p_{i}$ as $\varepsilon \rightarrow 0$. Moreover, the eigenvalues of the matrix $\left[D_{\mathbf{y}} g\left(p_{1}\right)\right]$ are 1 and $3 / 4$ and the eigenvalues of the matrices $\left[D_{\mathbf{y}} g\left(p_{2}\right)\right]$ and $\left[D_{\mathbf{y}} g\left(p_{3}\right)\right]$ are $(1 \pm \sqrt{7}) / 2$. Thus the $\mathrm{SE} a_{1}$ system has three periodic orbits bifurcating from the origin, one of them a repeller and a pair of periodic orbits of saddle type.

Going back to the initial variables of the $\mathrm{SE} a_{1}$ system, we get that, for $a>0$ sufficiently small, such system has three periodic orbits of period near to $2 \pi$, the first is given by $\gamma_{1}(t)=\left(x_{1}(t), y_{1}(t), z_{1}(t)\right)$, where

$$
x_{1}(t)=-\varepsilon+\mathcal{O}\left(\varepsilon^{2}\right), \quad y_{1}(t)=\mathcal{O}\left(\varepsilon^{2}\right), \quad z_{1}(t)=\mathcal{O}\left(\varepsilon^{2}\right),
$$

which is a repeller, and the other two are given by $\gamma_{2,3}=\left(x_{2,3}(t), y_{2,3}(t), z_{2,3}(t)\right)$, where

$$
\begin{aligned}
& x_{2,3}(t)=2( \pm \sqrt{3} \cos \theta+1) \varepsilon+\mathcal{O}\left(\varepsilon^{2}\right), \\
& y_{2,3}(t)= \pm 2 \sqrt{3} \varepsilon \sin \theta+\mathcal{O}\left(\varepsilon^{2}\right), \\
& z_{2,3}=\mp 2 \sqrt{3} \varepsilon \cos \theta+\mathcal{O}\left(\varepsilon^{2}\right),
\end{aligned}
$$

which are a pair of periodic orbits of saddle type.

Following the same steps and doing the suitable changes of variables it can be shown that the same results are valid for the other 22 differential systems of Tables 1, 2 and 3. We give explicit expressions for the change of variables needed to prove Theorem 1 for the $\mathrm{SE} a_{2}-\mathrm{SE} a_{23}$ systems in the Appendix, with some pertinent comments.

\section{On the Formation of the Hidden Chaotic Attractors}

Periodic orbits in chaotic differential systems are important dynamical elements in the formation of chaotic attractors. This was exhaustively studied in the case of self-exited attractors with the well-known period-doubling route to chaos and it also seems to happen in the case of the hidden attractors of these 23 differential systems here studied. In [3], for example, the authors showed that the hidden chaotic attractors in some NE systems (chaotic systems having no equilibrium points) are formed by a period-doubling of periodic orbits which bifurcate from a zero-Hopf equilibrium point at the origin.

In [17] the authors stated that the differential systems studied by them ( $\mathrm{SE} a_{1}-\mathrm{SE} a_{23}$ systems in Tables 1,2 and 3 with $\left.a=1\right)$ appear to approach 
chaos through a succession of period-doubling of periodic orbits. Here, by numerical simulations, we observed that this phenomenon also occurs for all the twenty three classes of differential systems given in Tables 1, 2 and 3, as stated in the conjecture, when the parameter $a$ varies. In order to avoid a long list of figures, we illustrate the formation of hidden chaotic attractors in $\mathrm{SE} a_{1}$ and $\mathrm{SE} a_{11}$ systems.

In Figures 1 and 2 are shown the formation of the hidden chaotic attractors in $\mathrm{SE} a_{1}$ and $\mathrm{SE} a_{11}$ systems as the parameter value $a$ tends to 1. Note that in both cases the formation of the hidden attractor begins with a periodic orbit which doubles period confirming the period-doubling route to chaos.

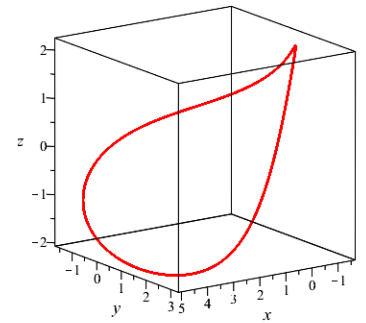

$a=0.97$

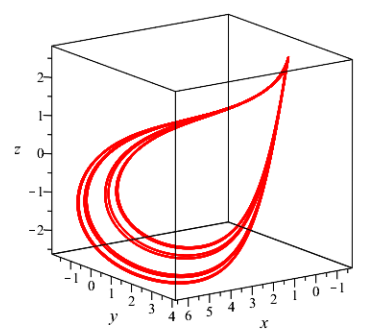

$a=0.99$

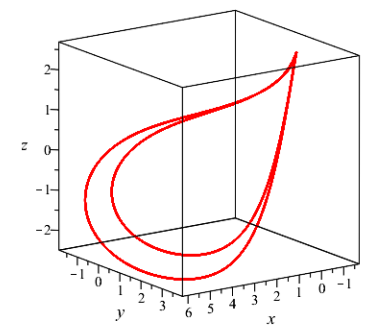

$a=0.987$

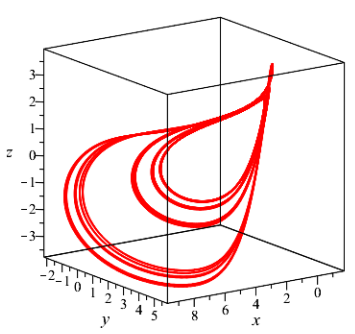

$a=1$

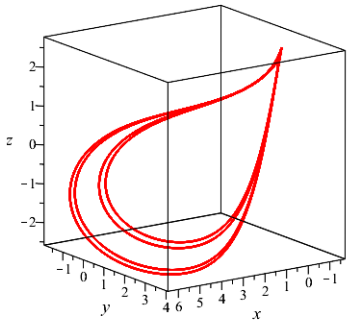

$a=0.989$

Figure 1. Orbit of the $\mathrm{SE} a_{1}$ system with initial condition $(4 a,-2 a, 0)$ for different values of the parameter $a$. This orbit is in the hidden chaotic attractor when $a=1$.

\section{Concluding Remarks}

In this paper we provide the expression of twenty three classes of quadratic differential systems in $\mathbb{R}^{3}$ depending on a real parameter $a$ which encompass the chaotic differential systems having hidden attractors studied in [17]. By using the averaging theory of first order presented in Section 2, we proved, in Section 3, that three periodic orbits, one of them being unstable and 


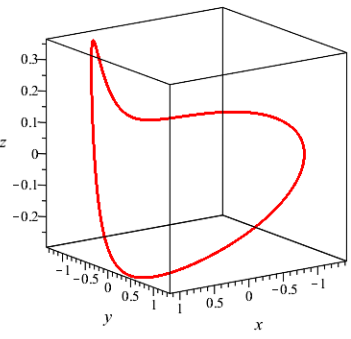

$a=0.72$

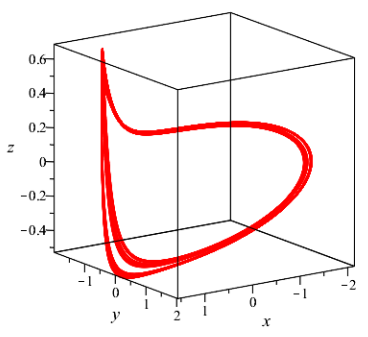

$a=0.962$

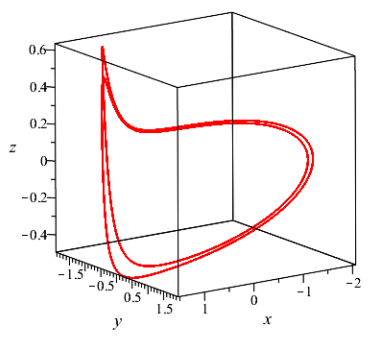

$a=0.94$

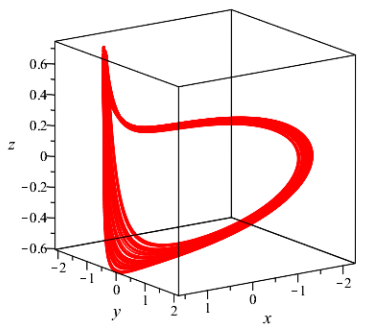

$a=1$

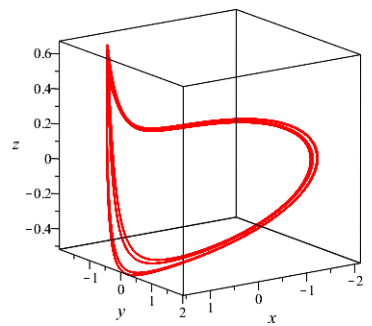

$a=0.955$

Figure 2. Orbit of the $\mathrm{SE} a_{11}$ system with initial condition $(-2 a, 0,0.1 a)$ for different values of the parameter $a$. This orbit is in the hidden chaotic attractor when $a=1$.

the other two forming a pair of periodic orbits of saddle type, bifurcate from a non-isolated zero-Hopf equilibrium point located at origin, when the parameter $a$ vary from $a=0$ to $a>0$ sufficiently small.

Doing numerical simulations we observed that the hidden chaotic attractors of all twenty three classes of differential systems given in Tables 1, 2 and 3 are formed by a period-doubling of periodic orbits. In Section 4 we presented the formation of the hidden attractor in the $\mathrm{SE} a_{1}$ and the $\mathrm{SE} a_{11}$ systems in order to illustrate this fact.

In this way, the existence of periodic orbits in the $\mathrm{SE} a_{1}-\mathrm{SE} a_{23}$ systems play an important role in the occurrence of chaotic behavior evidencing the importance of the zero-Hopf bifurcation stated by Theorem 1. Moreover, the existence of periodic orbits bifurcating from zero-Hopf equilibrium points and period-doubling route to chaos was also observed in $[3,4,16]$ for chaotic differential systems having no equilibrium points (the NE systems). Then, the occurrence of zero-Hopf bifurcation and the formation of hidden chaotic attractors seems to be strictly related. 


\section{REFERENCES}

[1] Abdolmohammadi, H.R., Khalaf, A.J.M., Panahi, S., Rajagopal, K., Pham, V.T., JAFARI, S. A new 4 d chaotic system with hidden attractor and its engineering applications: analog circuit design and field programmable gate array implementation. Pramana-J Phys 90 (2018), 1-7.

[2] Akgul, A., Calgan, H., Koyuncu, I., Penlivan, I., Istanbullu, A. Chaosbased engineering applications with a $3 D$ chaotic system without equilibrium points. Nonlinear Dyn, 84 (2016), 481-495.

[3] CÂndido, M.R., Llibre, J. Zero-Hopf bifurcations in 3-dimensional differential systems with no equilibria. Math Comput Simulat 151 (2018), 54-76.

[4] Carvalho, T., Euzébio, R., Llibre, J., Tonon, D.J. Detecting periodic orbits in some $3 D$ chaotic quadratic polynomial differential systems. Discrete Contin. Dyn. Syst. Ser. B 21 (2016), 1-11.

[5] ÇavuşoĞlu, Ü., Panahi, S., A Akgül, A., Jafari, S., KaÇar, S. A new chaotic system with hidden attractor and its engineering applications: analog circuit realization and image encryption. Analog Integr Circuits Signal Process 26 (2018), 1-15.

[6] Chua, L.O. Chua's circuit. An overview ten years later. J Circuits Syst Comput 4 (1994), 117-159.

[7] Jafari, S., Sprott, J.C. Simple chaotic flows with a line equilibrium. Chaos Solitons Fractals 57 (2013), 79-84.

[8] Jafari, S., Sprott, J.C., Golpayegani, S.M.R.H. Elementary quadratic chaotic flows with no equilibria. Phys Lett A 377 (2013), 699-702.

[9] Jafari, S., Sprott, J.C., Pham, V.T., Volos, C., Li, C. Simple chaotic 3D flows with surfaces of equilibria. Nonlinear Dyn 86 (2016), 1349-1358.

[10] Kingni, S.T., Jafari, S., Simo, H., WoAfo, P. Three-dimensional chaotic autonomous system with only one stable equilibrium: analysis, circuit design, parameter estimation, control, synchronization and its fractional-order form. Eur Phys J Plus 129 (2014), 76.

[11] Kuznetsov, N.V., Leonov, G.A., Vagaitsev, V.I. Analytical-numerical method for attractor localization of generalized Chua's system. IFAC Proc 43 (2010), 29-33.

[12] Leonov, G.A., Kuznetsov, N.V., Mokaev, T.N. Homoclinic orbits, and selfexcited and hidden attractors in a Lorenz-like system describing convective fluid motion. Eur Phys J 224 (2015), 1421-1458.

[13] Leonov G.A., Kuznetsov N.V., Vagaitsev V.I. Localization of hidden Chua's attractors. Phys Lett A 375 (2011), 2230-2233

[14] Li, C., Sprott, J.C. Coexisting hidden attractors in a 4-D simplified Lorenz system. Int J Bifurcat Chaos 24 (2014), 1450034 (12 pages).

[15] Lorenz, E.N. Deterministic nonperiodic flow. J.Atmos. Sci. 20 (1963), 130-141.

[16] Messias, M., Reinol, A.C. On the existence of periodic orbits and KAM tori in the Sprott A system: a special case of the Nosé-Hoover oscillator. Nonlinear Dyn 92 (2018), 1287-1297.

[17] Molaie, M., Jafari, S., Sprott, J. C., Golpayegani, S. M. R. H. Simple chaotic flows with one stable equilibrium. Int. J. Bifurcat. Chaos 23 (2013), 1350188 (7 pages).

[18] Rössler, O.E. An equation for continuous chaos. Phys Lett A 57 (1976), 397-398.

[19] Sanders, J.A., Verhulst, F., Murdock, J.: Averaging Methods in Nonlinear Dynamical Systems. Springer, New York, 2007.

[20] Sparrow, C. The Lorenz Equations: Bifurcations, Chaos and Strange Attractors. Springer, New York, 1982. 
[21] Vaidyanathan, S., Sambas, A., Mamat, M., Sanjaya, W.M. A new threedimensional chaotic system with a hidden attractor, circuit design and application in wireless mobile robot. Arch Control Sci 27 (2017), 541-554.

[22] Wang, Z., Cang, S., Ochola, E.O., Sun, Y. A hyperchaotic system without equilibrium. Nonlinear Dyn 69 (2012), 531-537.

[23] Wang, X., Chen, G. A chaotic system with only one stable equilibrium. Commun Nonlinear Sci 17 (2012), 1264-1272.

[24] Wei, Z., Pehlivan, I. Chaos, coexisting attractors, and circuit design of the generalized Sprott C system with only two stable equilibria. Optoelectron Adv Mat 6 (2012), 742-745.

[25] WeI, Z., ZhANG, W. Hidden hyperchaotic attractors in a modified Lorenz-Stenflo system with only one stable equilibrium. Int J Bifurcat Chaos 24 (2014), 1450127 (14 pages).

\section{Appendix: Complement of the PRoOf of Theorem 1}

In Section 3 we give a detailed proof of Theorem 1 for the $\mathrm{SE} a_{1}$ system. By following the same steps and doing the appropriated changes of variables it can be proved that the same results are valid for the $\mathrm{SE} a_{2}-\mathrm{SE} a_{23}$ systems of Tables 1, 2 and 3. For the sake of completeness, we provide some information about the proof of Theorem 1 for $\mathrm{SE} a_{2}-\mathrm{SE} a_{23}$ systems by considering the following steps:

i) For $a=0$ the $x$-axis is filled up of equilibrium points for $\mathrm{SE} a_{2}-$ $\mathrm{SE} a_{6}$ and $\mathrm{SE} a_{18}-\mathrm{SE} a_{23}$ systems, the $y$-axis is filled up of equilibrium points for $\mathrm{SE} a_{14}-\mathrm{SE} a_{16}$ systems, the $z$-axis is filled up of equilibrium points for $\mathrm{SE} a_{7}-\mathrm{SE} a_{13}$ systems and the straight line $y=x$ is a curve of equilibrium points for $\mathrm{SE} a_{17}$ system. In all systems the straight line of equilibrium points, in a neighborhood of the origin, is formed by two branches of foci with opposite stability connected by a nonisolated zero-Hopf equilibrium point at the origin.

ii) In order to put the systems in the normal form of the averaging theorem, first consider the linear change of variables $(x, y, z) \rightarrow(u, v, w)$, where

$$
\begin{array}{llll}
x=u+w, & y=v, & z=-u, & \text { for } \mathrm{SE} a_{2}-\mathrm{SE} a_{6} \text { systems, } \\
x=u, & y=v, & z=w, & \text { for } \mathrm{SE} a_{7}-\mathrm{SE} a_{13} \text { systems, } \\
x=u, & y=u-v+w, & z=v, & \text { for } \mathrm{SE} a_{14} \text { system, } \\
x=u, & y=u-v+w, & z=-v, & \text { for } \mathrm{SE} a_{15} \text { system, } \\
x=u, & y=-u+v+w, & z=-v, & \text { for } \mathrm{SE} a_{16} \text { system, } \\
x=2 v+w, & y=u+v+w, & z=-2 u, & \text { for } \mathrm{SE} a_{17} \text { system, } \\
x=2 v+w, & y=-u+v, & z=-2 u, & \text { for } \mathrm{SE} a_{18} \text { system, } \\
x=-2 v+w, & y=u-v, & z=2 u, & \text { for } \mathrm{SE} a_{19} \text { system, } \\
x=-2 v+w, & y=-u+v, & z=2 u, & \text { for } \mathrm{SE} a_{20}-\mathrm{SE} a_{22} \text { systems, } \\
x=2 v+w, & y=-u+v, & z=2 u, & \text { for } \mathrm{SE} a_{23} \text { system. }
\end{array}
$$

After these changes of variables the linear part at the origin of the $\mathrm{SE} a_{2}-\mathrm{SE} a_{23}$ systems when $a=0$ is in the real Jordan normal form. 
iii) Write the obtained systems in the cylindrical coordinates $(r, \theta, w)$, where $u=r \cos \theta$ and $v=\sin \theta$.

$i v$ ) Introduce the variable $\varepsilon>0$ into the systems by considering $a=\varepsilon$ and doing a rescaling of the variables through the change of coordinates $(r, \theta, w) \rightarrow(R, \theta, W)$, where $r=\varepsilon R$ and $w=\varepsilon W$.

$v$ ) Taking $\theta$ as the new independent variable and doing the Taylor expansion in the variable $\varepsilon$ at $\varepsilon=0$ up to order 2 we put the systems in the form of Theorem 2 .

vi) Using the notation of Theorem 2 and solving the equation $g(\mathbf{y})=0$ we get the real solutions $p_{i}=\left(R_{i}, W_{i}\right)$, for $i=1,2,3$, where

$$
\begin{array}{lll}
p_{1}=(0,1), & p_{2,3}=\left( \pm \frac{\sqrt{1265}}{11},-\frac{1}{22}\right), & \text { for } \mathrm{SE} a_{2} \text { system }, \\
p_{1}=\left(0,-\frac{5}{17}\right), & p_{2,3}=\left( \pm \frac{2 \sqrt{127}}{5}, \frac{6}{5}\right), & \text { for } \mathrm{SE} a_{3} \text { system }, \\
p_{1}=(0,-1), & p_{2,3}=\left( \pm \frac{4 \sqrt{15}}{5}, \frac{7}{5}\right), & \text { for } \mathrm{SE} a_{4} \text { system }, \\
p_{1}=\left(0,-\frac{27}{10}\right), & p_{2,3}=\left( \pm \frac{3 \sqrt{30}}{5}, 0\right), & \text { for } \mathrm{SE} a_{5} \text { system }, \\
p_{1}=(0,-1), & p_{2,3}=\left( \pm \frac{\sqrt{22}}{11},-\frac{10}{11}\right), & \text { for } \mathrm{SE} a_{6} \text { system }, \\
p_{1}=\left(0,-\frac{1}{2}\right), & p_{2,3}=( \pm \sqrt{2}, 0), & \text { for } \mathrm{SE} a_{7} \text { system }, \\
p_{1}=\left(0,-\frac{1}{10}\right), & p_{2,3}=\left( \pm \frac{\sqrt{6}}{3}, 0\right), & \text { for } \mathrm{SE} a_{8} \text { system }, \\
p_{1}=\left(0,-\frac{3}{20}\right), & p_{2,3}=\left( \pm \frac{\sqrt{15}}{5}, 0\right), & \text { for } \mathrm{SE} a_{9} \text { system }, \\
p_{1}=\left(0,-\frac{1}{20}\right), & p_{2,3}=\left( \pm \frac{\sqrt{5}}{5}, 0\right), & \text { for } \mathrm{SE} a_{10} \text { system }, \\
p_{1}=\left(0,-\frac{1}{12}\right), & p_{2,3}=( \pm \sqrt{2}, 0), & \text { for } \mathrm{SE} a_{11} \text { system },
\end{array}
$$




$$
\begin{aligned}
& p_{1}=\left(0,-\frac{1}{66}\right), \quad p_{2,3}=( \pm \sqrt{2}, 0), \quad \text { for } \mathrm{SE} a_{12} \text { system } \\
& p_{1}=\left(0,-\frac{10}{49}\right), \quad p_{2,3}=( \pm \sqrt{5}, 0), \quad \text { for } \mathrm{SE} a_{13} \text { system } \\
& p_{1}=\left(0,-\frac{7}{10}\right), \quad p_{2,3}=\left( \pm \frac{\sqrt{10}}{5},-\frac{1}{2}\right), \quad \text { for } \mathrm{SE} a_{14} \text { system } \\
& p_{1}=\left(0,-\frac{10}{9}\right), \quad p_{2,3}=\left( \pm \frac{\sqrt{190}}{20},-\frac{9}{10}\right), \quad \text { for } \mathrm{SE} a_{15} \text { system } \\
& p_{1}=\left(0,-\frac{1}{7}\right), \quad p_{2,3}=( \pm 4 \sqrt{6},-7), \quad \text { for } \mathrm{SE} a_{16} \text { system } \\
& p_{1}=\left(0, \frac{57}{310}\right), \quad p_{2,3}=\left( \pm \frac{3 \sqrt{39590}}{740}, \frac{21}{148}\right), \text { for } \mathrm{SE} a_{17} \text { system } \\
& p_{1}=\left(0,-\frac{10}{21}\right), \quad p_{2,3}=\left( \pm \frac{\sqrt{14685}}{165},-\frac{1}{11}\right), \quad \text { for SE } a_{18} \text { system } \\
& p_{1}=\left(0,-\frac{3}{10}\right), \quad p_{2,3}=\left( \pm \frac{\sqrt{30}}{10}, 0\right), \quad \text { for } \mathrm{SE} a_{19} \text { system } \\
& p_{1}=\left(0,-\frac{1}{11}\right), \quad p_{2,3}=\left( \pm \frac{\sqrt{13}}{78},-\frac{1}{12}\right), \quad \text { for } \mathrm{SE} a_{20} \text { system } \\
& p_{1}=\left(0, \frac{10}{71}\right), \quad p_{2,3}=\left( \pm \frac{\sqrt{569}}{70}, \frac{61}{490}\right), \quad \text { for SE } a_{21} \text { system } \\
& p_{1}=\left(0,-\frac{3}{20}\right), \quad p_{2,3}=\left( \pm \frac{\sqrt{10}}{10},-\frac{1}{10}\right), \quad \text { for SE } a_{22} \text { system } \\
& p_{1}=\left(0, \frac{1}{2}\right), \quad p_{2,3}=\left( \pm \frac{2 \sqrt{3}}{3}, \frac{3}{10}\right), \quad \text { for SE } a_{23} \text { system, }
\end{aligned}
$$

which satisfy $\operatorname{det}\left[D_{\mathbf{y}} g\left(p_{1}\right)\right] \cdot \operatorname{det}\left[D_{\mathbf{y}} g\left(p_{2}\right)\right] \cdot \operatorname{det}\left[D_{\mathbf{y}} g\left(p_{3}\right)\right] \neq 0$. By Theorem 2 , for $\varepsilon>0$ sufficiently small, the $\mathrm{SE} a_{2}-\mathrm{SE} a_{23}$ systems have the periodic solutions $\phi_{i}(\theta, \varepsilon)=\left(R_{i}(\theta, \varepsilon), Z_{i}(\theta, \varepsilon)\right)$, for $i=1,2,3$, such that $\phi_{i}(0, \varepsilon) \rightarrow p_{i}$ as $\varepsilon \rightarrow 0$. Moreover, the eigenvalues of the matrix $\left[D_{\mathbf{y}} g\left(p_{1}\right)\right]$ are real and positive and the eigenvalues of the matrices $\left[D_{\mathbf{y}} g\left(p_{2}\right)\right]$ and $\left[D_{\mathbf{y}} g\left(p_{3}\right)\right]$ are real with different signs.

Therefore, $\mathrm{SE} a_{2}-\mathrm{SE} a_{23}$ systems have three periodic orbits bifurcating from the origin, one of them a repeller and a pair of periodic orbits of saddle type. 
1 Departament de Matemàtiques, Universitat Autònoma de Barcelona UAB, 08193 Bellaterra, Barcelona, Catalonia, Spain.

Email address: jllibre@mat.uab.cat

${ }^{2}$ Departamento de Matemática e ComputaÇão, Universidade Estadual Paulista - FCt/Unesp, Presidente Prudente, SÃo Paulo, Brazil.

Email address: marcelo@fct.unesp.br

${ }^{3}$ Departamento Acadêmico de Matemática, Universidade Tecnológica Federal do Paraná - UTFPR-CP, Cornélio Procópio, Paraná, Brazil.

Email address: alissoncarv@gmail.com 